\title{
Nonradial neutrino emission upon black hole formation in core collapse supernovae
}

\author{
Jia-Shian Wang $\odot$ and Jeff Tseng $\odot^{*}$ \\ Department of Physics, Oxford University, Oxford OX1 3RH, United Kingdom \\ Samuel Gullin and Evan P. O'Connor@ \\ The Oskar Klein Centre, Department of Astronomy, Stockholm University, \\ AlbaNova, SE-106 91 Stockholm, Sweden
}

(Received 30 September 2021; accepted 7 October 2021; published 12 November 2021)

\begin{abstract}
Black hole formation in a core-collapse supernova is expected to lead to a distinctive, abrupt drop in neutrino luminosity due to the engulfment of the main neutrino-producing regions as well as the strong gravitational redshift of those remaining neutrinos which do escape. Previous analyses of the shape of the cutoff have focused on specific trajectories or simplified models of bulk neutrino transport. In this article, we integrate over simple null geodesics to investigate potential effects on the cutoff profile of including all neutrino emission angles from a collapsing surface in the Schwarzschild metric, and from a contracting equatorial mass ring in the Kerr metric. We find that the nonradial geodesics contribute to a softening of the cutoff in both cases. In addition, extreme rotation introduces significant changes to the shape of the tail which may be observable in future neutrino detectors, or combinations of detectors.
\end{abstract}

DOI: 10.1103/PhysRevD.104.104030

\section{INTRODUCTION}

The conventional picture of a core-collapse supernova (CCSN) begins with a large stellar progenitor, with a zeroage main sequence (ZAMS) mass greater than approximately 10 solar masses $M_{\odot}$, collapsing to a neutron star. In the process, the vast majority of the energy is released in a burst of neutrinos which escapes the conflagration well before any electromagnetic radiation. It is expected that neutrino and dark matter detectors will record an abnormally high rate of neutrino events hours before the supernova becomes visible in the sky; this lead time is the motivation behind the Supernova Neutrino Early Warning System (SNEWS) [1], which should play a key role in observing the next galactic CCSN through its neutrino, gravitational, and electromagnetic "messengers."

For some stellar progenitors (though the question of which ones remains debated [2]), the outcome may be very different, as the proto-neutron star (PNS) may itself collapse into a black hole. In such cases, the neutrino (and gravitational wave) signal may not be followed by a traditional, visible electromagnetic signal. One distinctive indication of such an end result is an abrupt cutoff in the neutrino luminosity. This cutoff is the result of the black hole engulfing the neutrino-producing regions of the PNS, and the gravitational redshift of those which just manage to escape.

* Corresponding author. jeff.tseng@physics.ox.ac.uk
The shape of the cutoff was estimated first based on photons emitted from a free falling, nonrotating mass shell. A conventional treatment of radial trajectories gives an exponential decline with a time constant of $4 M$, where $M$ is the mass of the black hole [3]. However, late-time behavior is expected to be dominated by neutrinos trapped for an extended period around unstable circular orbits near a critical radius $3 M$, resulting in a time constant of $3 \sqrt{3} M[4,5]$. In a CCSN, of course, the photons will be absorbed well before they escape. Instead, the cutoff would only be evident in the neutrinos escaping from near the growing black hole $[6,7]$.

Because of uncertainties in the structure of the PNS, it is unclear how much of the PNS is involved in the creation of the black hole. It is likely, however, that the event horizon is formed initially below the surface of the PNS, leaving the most fertile neutrino production layers outside. If this is the case, those outside layers will still be emitting neutrinos in all directions, rather than radially, before they themselves fall behind the event horizon. Indeed, it was suggested in [8] that the radial estimate is expected to be an underestimate, and that a full treatment would require detailed ray-tracing through a highly curved spacetime.

Nonetheless, the neutrino cutoff is a compelling feature of black hole formation within a CCSN because of its simplicity, distinctiveness, and the fact that it could reflect events occurring deep within one of the most turbulent and violent phenomena known in the universe. It can also add statistical power to triangulating the direction of the CCSN if observed in several detectors [9-11]. Models of this 
phenomenon are usually the domain of computationally intensive hydrodynamic simulations which of necessity simplify neutrino transport to varying degrees, and approximate or incorporate full general relativity (see, for example, $[12,13])$. In many cases, however, black hole formation heralds the end of validity of such simulations.

In this article, we investigate effects of nonradial geodesics on the neutrino cutoff for both nonrotating and rotating black holes. These idealized trajectories are clearly a drastic simplification within a complex domain, but as a toy model can highlight broad features and be a useful check of more detailed simulations. Null geodesics from collapsing stars have been investigated in $[5,14,15]$ for photons, as well as in $[16,17]$ for neutrinos, though in contexts somewhat different from modern models of a CCSN.

This article is organized as follows: we consider the appropriateness of these simple trajectories to the core collapse scenario in Sec. II. Section III concerns time delays in the nonrotating Schwarzschild metric. The Kerr metric, for a rotating black hole, is considered in Sec. IV. The results are summarized and discussed in Sec. V.

\section{NEUTRINOS IN AN EVOLVING SPACETIME}

The main source of neutrinos in a CCSN is its hot, dense core. In the initial stages of the core collapse, the core undergoes rapid neutronization, leading to a burst of $\nu_{e}$. Later stages see the thermal production of all neutrino flavors within the coalescing PNS as it continues to accrete matter from the collapsing star. Two scenarios are envisaged which result in the formation of black hole [18]: in the first, the accretion compresses the PNS further until enough matter is compressed into a small enough radius that the geodesics of massless particles curve back on themselves, i.e., an event horizon is formed. In the second, a part of the PNS undergoes a nuclear phase transition, leading again to higher densities which form an event horizon.

In either case, it is worth noting that the formation of the event horizon, and therefore of the black hole itself, is in a sense a global observable irrelevant to a neutrino travelling nearby. Assuming that the neutrino can pass through the environment (a transition which is expected to occur at some point during PNS cooling), the neutrino is only sensitive to the local curvature, which is determined by how mass is distributed around it. As mass is redistributed around it, generally "inward" in a global sense, its geodesic bends accordingly. The only indication that a black hole has formed is that some (but by no means all) paths bend back on themselves so that they cannot escape.

Moreover, the timescale on which geodesics bend further is not altogether sudden. It is expected that mass accretion onto the PNS will be on the order of $1 M_{\odot} / \mathrm{s}$ by the time a black hole forms [13]. If we take this also as an estimate of how quickly the black hole grows, then its outermost circular orbit radius grows at around $3 M_{\odot} / \mathrm{s}$ (using units in which $G=c=1$ ), or perhaps more intuitively, on the order of kilometers per second. On the other hand, the speed of the neutrinos is nearly the speed of light, more than $10^{5}$ greater. From this perspective, the slow increase in the size of the black hole should not significantly affect the neutrino path.

\section{TIME DELAYS IN THE SCHWARZSCHILD GEOMETRY}

We first consider neutrinos being emitted (or undergoing their last scatter before escaping) from a shell of matter free-falling radially inward toward a nonrotating black hole. We start from the Schwarzschild metric in the standard coordinates $(t, r, \theta, \phi)$,

$$
\begin{aligned}
\mathrm{d} s^{2}= & -\left(1-\frac{2 M}{r}\right) \mathrm{d} t^{2}+\left(1-\frac{2 M}{r}\right)^{-1} \mathrm{~d} r^{2} \\
& +r^{2} \mathrm{~d} \theta^{2}+r^{2} \sin ^{2} \theta \mathrm{d} \phi^{2}
\end{aligned}
$$

where $M$ is the mass of the black hole. The geodesic solutions are well known, but we summarize them here.

Since the metric is spherically symmetric, the geodesics lie in an equatorial plane, for which we choose $\theta=\frac{\pi}{2}$. The conserved quantities associated with the metric's two Killing vectors are

$$
\begin{gathered}
E=-g_{\alpha \beta}\left(\frac{\partial}{\partial t}\right)^{\alpha}\left(\frac{\partial}{\partial \tau}\right)^{\beta}=C(r) \frac{\mathrm{d} t}{\mathrm{~d} \tau} \\
L=g_{\alpha \beta}\left(\frac{\partial}{\partial \phi}\right)^{\alpha}\left(\frac{\partial}{\partial \tau}\right)^{\beta}=r^{2} \frac{\mathrm{d} \phi}{\mathrm{d} \tau}
\end{gathered}
$$

where $\tau$ is the proper time of the neutrino, and

$$
C(r)=\left(1-\frac{2 M}{r}\right) \text {. }
$$

For massive particles, $E$ and $L$ can be interpreted as energy and angular momentum per unit mass.

\section{A. Neutrino propagation time}

The emitting matter starts from rest at an initial radius $r_{0}$, from which it free-falls. In this case, $L=0$ and we have

$$
\left(\frac{\mathrm{d} r}{\mathrm{~d} t}\right)^{2}=\left(1-\frac{C}{C_{0}}\right) C^{2},
$$

where $C_{0} \equiv C\left(r_{0}\right)$. For the paths of the nearly massless neutrinos, we use null geodesics (in which case $\tau$ is technically the affine parameter rather than the proper time). The geodesics are solutions of the equation

$$
\left(\frac{\mathrm{d} r}{\mathrm{~d} t}\right)^{2}=\left(1-\frac{2 M}{r}\right)^{2}\left(1-\frac{b^{2}}{r^{2}}\left(1-\frac{2 M}{r}\right)\right),
$$


where $b \equiv L / E$ is the impact parameter as observed by a distant observer.

In our model, neutrinos are emitted isotropically in the frame comoving with the emitter, which we will refer to as the "free-falling" $(F F)$ frame. The emission direction in the free-falling frame is directly related to the impact parameter defined above via the frame of an observer static at the radius at which the emission occurs. We denote this "static" frame as $S$.

In the $S$ frame, the (inward) speed of the emitting surface is

$$
\beta_{S}=\sqrt{1-\frac{C}{C_{0}}}
$$

The radial velocity of the neutrino upon emission is

$$
\left(\frac{\mathrm{d} r}{\mathrm{~d} t}\right)_{S}=\cos \psi_{S}=\sigma_{r} \sqrt{1-\frac{b^{2}}{r^{2}}\left(1-\frac{2 M}{r}\right)},
$$

where $\psi_{S}$ is the angle relative to the radial outward direction as measured in the static frame; and $\sigma_{r}$ is +1 for an outward trajectory, and -1 for an inward trajectory, again as measured in the static frame. The radial velocity can be identified simply as $\cos \psi_{S}$, since for null geodesics the total velocity is always $c=1$. This identification also applies to all other frames.

The corresponding emission angle $\psi_{F F}$ in the $F F$ frame is then related to $\psi_{S}$ through velocity addition,

$$
\left(\frac{\mathrm{d} r}{\mathrm{~d} t}\right)_{F F}=\cos \psi_{F F}=\frac{\cos \psi_{S}+\beta_{S}}{1+\beta_{S} \cos \psi_{S}} .
$$

In this way, a value of $b$ can be calculated given an emission angle $\psi_{F F}$. From Eqs. (8) and (9), one can clearly see that radial emission corresponds to $b=0$.

Integrating Eq. (6) results in the following expression for the travel time along the geodesic:

$$
T\left(b, r_{*} ; r_{E}\right)=\int_{r_{*}}^{r_{E}} \frac{r^{5 / 2} \mathrm{~d} r}{(r-2 M) \sqrt{r^{3}-b^{2}(r-2 M)}},
$$

where $r_{*}$ is the emission radius, and $r_{E}$ is the distance to the Earth. This expression can be simplified for the case of radial emission

$$
\begin{aligned}
T(b & \left.=0, r_{*} ; r_{E}\right)=\int_{r_{*}}^{r_{E}} \frac{r \mathrm{~d} r}{r-2 M} \\
& =\left(r_{E}-r_{*}\right)+2 M \ln \left(\frac{r_{E}-2 M}{r_{*}-2 M}\right) .
\end{aligned}
$$

Figure 1 shows two cases to consider when calculating the time delay of a neutrino. For a neutrino emitted in an

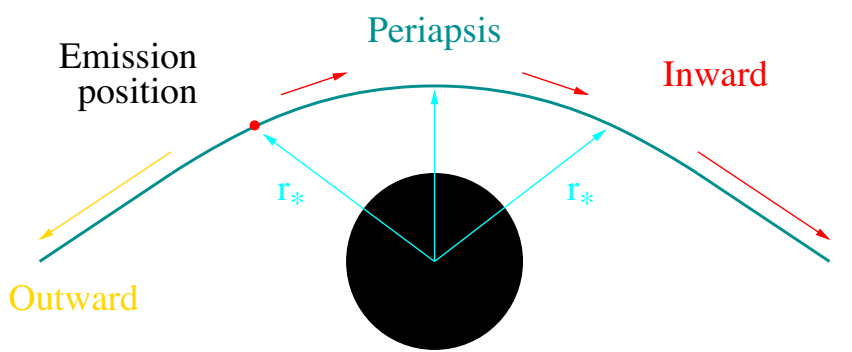

FIG. 1. Inward and outward trajectories for neutrinos emitted outside the photon sphere. Outward trajectories escape directly, while inward trajectories reach periapsis before joining the outward trajectory.

outward direction (in the static frame $S$ ), the time delay relative to the travel time of radial emission from the initial radius is

$$
\Delta T_{+}\left(b, r_{*}\right)=T\left(b, r_{*} ; r_{E}\right)-T\left(0, r_{0} ; r_{E}\right) .
$$

If, on the other hand, the neutrino is emitted in an inward direction, it first acquires a Shapiro-like time delay as it passes the periapsis. The travel time is then

$$
\Delta T_{-}\left(b, r_{*}\right)=2 T\left(b, r_{p} ; r_{*}\right)+\Delta T_{+}\left(b, r_{*}\right),
$$

where $r_{p}$ is the periapsis distance

$$
r_{p}=\frac{2 b}{\sqrt{3}} \cos \left(\frac{1}{3} \arccos \left(-\frac{\sqrt{27} M}{b}\right)\right) .
$$

The observation time $t_{E}$ of a neutrino emitted at time $t_{*}$ from the shell at radius $r_{*}$ can then be written as

$$
t_{E}=t_{*}+\Delta T_{ \pm}\left(b, r_{*}\right) .
$$

It is evident from the integrand in Eq. (10) that not all neutrinos escape to large distances. There are two conditions in which the integrand diverges: $r=2 M$ and $r^{3}=b^{2}(r-2 M)$. The first condition gives the event horizon, while the second condition defines the so-called "photon sphere" at $r=3 M$, for which paths with the "critical impact parameter" $b_{\text {crit }} \equiv 3 \sqrt{3} M$ follow an unstable circular orbit. Outside the photon sphere, all outward geodesics, along with inward geodesics with impact parameters satisfying

$$
b_{\text {crit }}<b \leq \sqrt{\frac{r^{3}}{r-2 M}},
$$

are able to reach infinity. Between the photon sphere and the event horizon, only outward geodesics with $b<b_{\text {crit }}$ reach infinity. Figure 2 shows these "escape cones" (the opposite of Chandrasekhar's "cones of avoidance" [19]). 


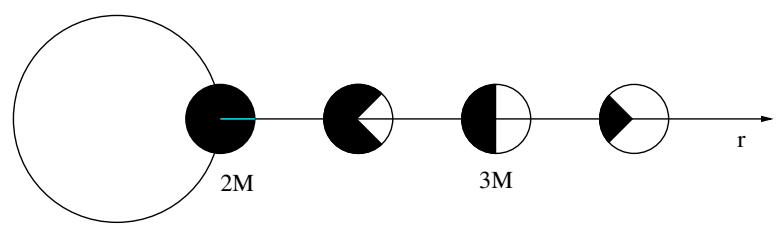

FIG. 2. Schematic view of the escape cones (unshaded region) for null geodesics in Schwarzschild spacetime. At $r_{*}=2 M$, only radial geodesics can escape. For $2 M<r_{*} \leq 3 M$, only outwardoriented geodesics are able to escape. Beyond $r_{*}=3 M$, some of the inward-oriented geodesics can also escape to infinity.

The dependence of the time delay on emission angle is shown in Fig. 3, for a surface initially at rest at radius $10 M$ and falling toward a black hole of mass $M=2.5 M_{\odot}$. Since $\psi_{F F}=0$ is defined as the outward radial direction, its time delay is zero by definition. The delay increases with deviation from the radial direction, and diverges as the periapsis radius approaches the photon sphere radius. Moreover, as the emitting shell of matter falls, its velocity increases and soon reaches speeds which are sizable fractions of $c$; in the given configuration, the emitters reach a free-fall speed of $0.31 c$ at radius $4.3 \mathrm{M}$, and pass the photon sphere in $0.55 \mathrm{~ms}$.

\section{B. Luminosity profile}

The cutoff profile of the luminosity as a function of observation time $t_{E}$ is governed by the number of neutrinos reaching the observer as well as by their redshift [5]

$$
\zeta\left(b, r_{*}, \sigma_{r}\right)=\frac{\nu_{E}}{\nu_{*}}=\sqrt{C} \times \frac{\sqrt{1-\beta_{S}^{2}}}{1+\beta_{S} \cos \psi_{S}},
$$

where $\nu_{*}$ and $\nu_{E}$ are the neutrino energies at emission and upon observation at the Earth. The first factor of Eq. (17)

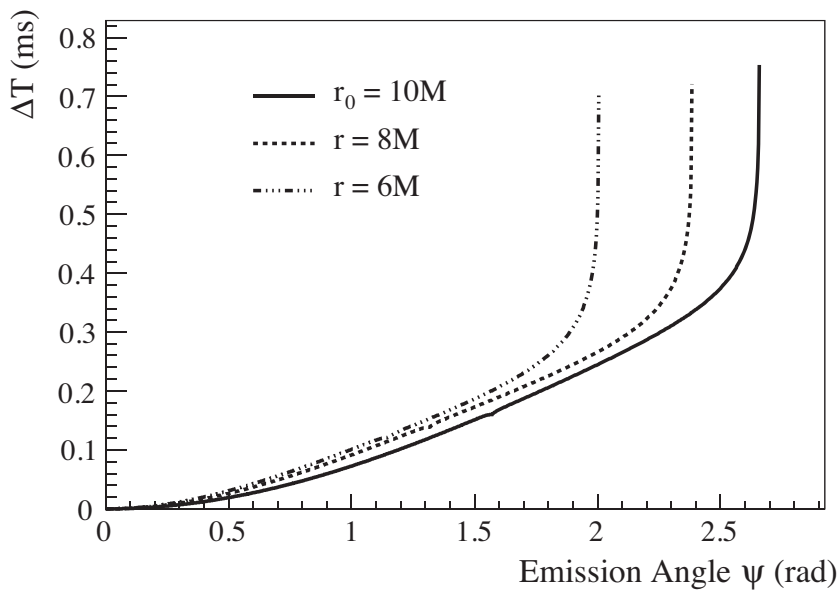

FIG. 3. Time delays, relative to that of the outward radial emission for a given radius, as a function of the emission angle in the $F F$ frame. The curves are shown for the initial radius $r_{0}=$ $10 M$ and for subsequent radii $8 M$ and $6 M$, for a Schwarzschild black hole with mass $2.5 M_{\odot}$. is the gravitational redshift, assuming $r_{E} \gg r_{*}$, and the second factor the Doppler shift. Figure 4(a) shows the redshift factor for outward radial emissions $(b=0)$ and "critical emissions" (those with $b=b_{\text {crit }}$ ) as a function of their observation times, relative to the arrival of the first neutrino from the shell's initial radius. The profiles take the form of exponentials with a possible offset,

$$
A e^{-t / \tau(t)}+B
$$

We refer to $\tau(t)$ as the "decay parameter" at time $t$, or as the "(decay) time constant" when it is indeed constant. The decay parameter is shown in Fig. 4(b). At late times, the radial redshift curve approaches a decay parameter value of $4 M$, consistent with the standard result [3]. The redshift curve for critical emissions, on the other hand, flattens out as the shell approaches the circular orbit at radius $3 M$; this feature is analogous to the "photon cloud" of [5]. If we subtract off the asymptotic offset, we are left with an exponential-like attenuation with a decay parameter which approaches $3 \sqrt{3} M$, which is the result of [4,5]. Inside radius $3 M$, the critical emissions fall outside the escape cone and do not reach a distant observer.

The energy contribution at observation time $t_{E}$ of neutrinos emitted at other angles from radius $r$ and time $t$, with impact parameter $b$, can be written as

$$
\mathrm{d} \epsilon\left(t_{E}\right)=\zeta\left(b, r, \sigma_{r}\right) \times \frac{L_{0} \cdot \mathrm{d} t}{4 \pi r^{2}} \times r^{2} \mathrm{~d} \Omega,
$$

where $\mathrm{d} \Omega$ is the solid angle element of the emitting shell, and $L_{0}$ is the total luminosity of the surface. It is
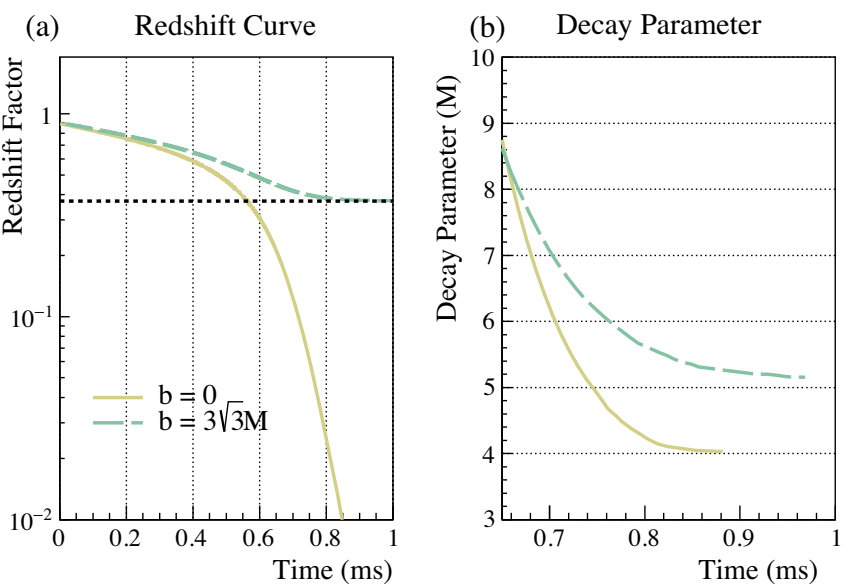

FIG. 4. (a) Redshift factor and (b) redshift decay parameter for outward radial emissions ( $b=0$, solid/yellow line) and critical emissions ( $b=b_{\text {crit }} \equiv 3 \sqrt{3} M$, dashed/green line) plotted against observation time for a $2.5 M_{\odot}$ Schwarzschild black hole. The curves are calculated for a shell falling from $10 M$ to $2.1 M$ (for $b=0$ ) or $3 M$ (for $b=b_{\text {crit }}$ ). The observation time is taken relative to the observation of the first neutrino received from the shell when it was at $10 \mathrm{M}$. The dashed black line in (a) is the redshift at the unstable circular orbit at $r=3 \mathrm{M}$. 


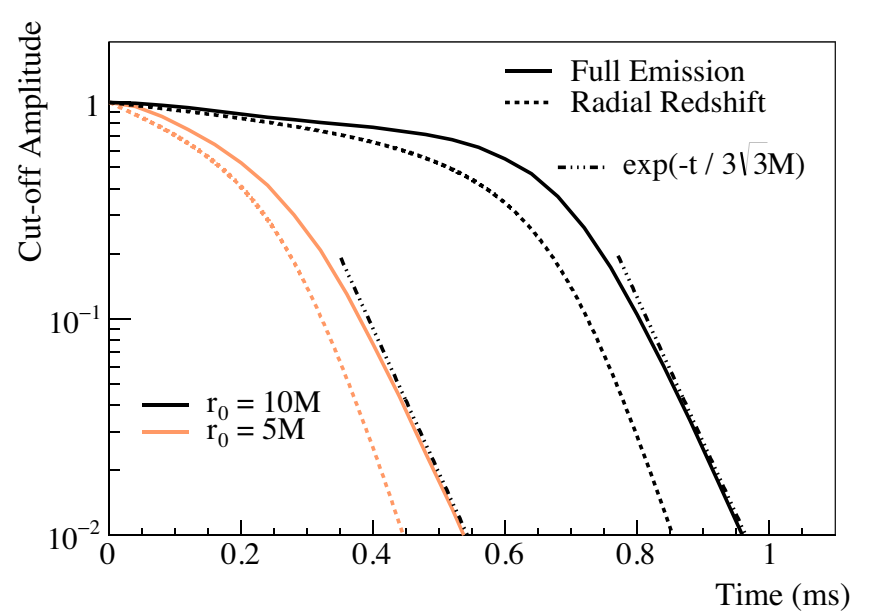

FIG. 5. Comparison of cutoff profiles for nonradial (solid) and radial (dotted) emissions for initial radii $r_{0}=10 M$ (black) and $5 M$ (orange). The profiles are normalized to 1 at $t_{E}=0$. The late time behaviors approach falling exponentials with time constant $3 \sqrt{3} M$, which are indicated with black dotted-dashed lines.

assumed that the total luminosity is constant throughout the collapse.

We evaluate the luminosity as a function of observed time using a simple ray-tracing Monte Carlo model. At each step in coordinate time $t$, we simulate a fixed number of isotropic neutrino emissions from the collapsing shell. The emission angle is used to calculate the impact parameter $b$, the corresponding observation time $t_{E}$ (if finite), and the redshift factor $\zeta$. Moreover, we simplify the simulation further in light of its spherical symmetry by simulating emissions from only one point on the shell, and counting neutrinos at radius $r_{E}$, regardless of where the neutrino intersects the outer sphere. The results are shown in Fig. 5 for initial radii $r_{0}=10 M$ and $5 M$ : there is a slow drop in the luminosity for several tenths of ms, followed by a steepening which rapidly approaches a decay parameter of $3 \sqrt{3} M$. The $3 \sqrt{3} M$ decay parameter thus characterizes much of the cutoff rather than only the very end, where dominance by critical emissions near $r=3 M$ has long been expected [8]. Moreover, it is evident that consideration of all emission directions softens the overall cutoff: Fig. 6, for example, shows that at all times, the decay parameter of the full emissions curve exceeds even that of critical emissions.

It should be noted that Fig. 5 shows the result of all the neutrinos emitted from the collapsing shell and escaping to large distances, i.e., the surrounding medium is transparent to the neutrinos. If, on the other hand, the inner medium is assumed to be completely opaque to neutrinos, then only those neutrinos emitted outwards in the free-falling frame $F F$ will escape. The cutoff profiles of this "opaque shell" scenario are shown in Fig. 7. The difference between the opaque shell scenario and that of allowing only outward emissions in the static $(S)$ frame is the effect of neutrinos

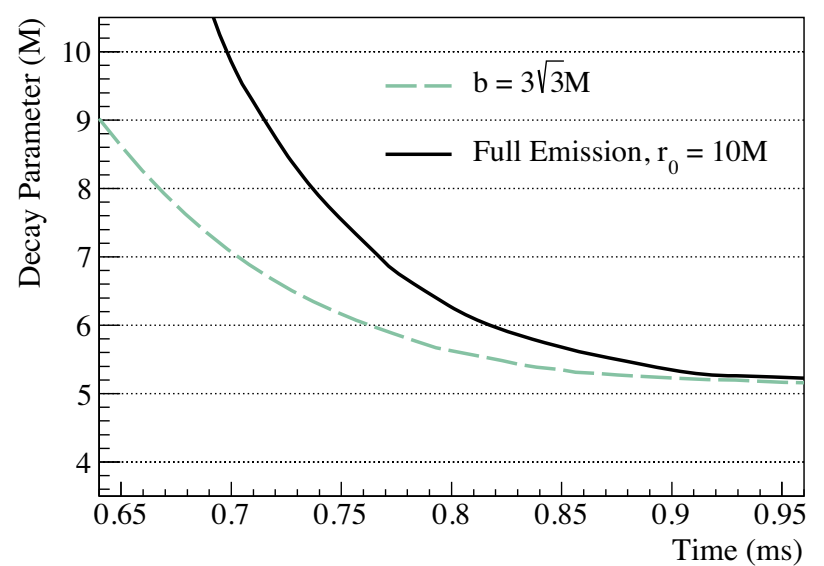

FIG. 6. The decay parameter for critical emissions $\left(b=b_{\text {crit }}\right)$ (dashed/green) and full emissions (solid/black), with initial radius $r_{0}=10 M$.

which appear in $S$ to be directed inward, but actually lag behind the collapsing shell and the opaque medium beneath it. In the end, the opaque shell scenario is a small modification on that of full emission, and only introduces minor changes to the decay timescale.

\section{Shells not in free fall}

The opaque shell is one starting point for introducing more realism into this toy model. For instance, we can reduce the shell's proper acceleration by a constant factor $f$ to mimic residual pressure support. The proper velocity will be

$$
\frac{\mathrm{d} r}{\mathrm{~d} \tau}=\sqrt{f\left(C_{0}-C\right)}
$$

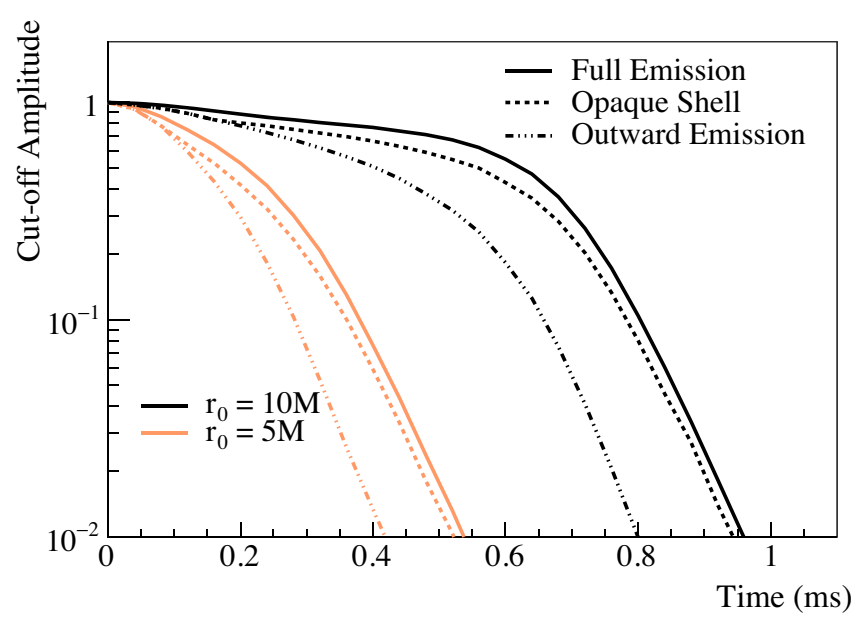

FIG. 7. Comparison of three different inward emission scenarios for initial radii $r_{0}=10 M$ (black) and $5 M$ (orange): transparent medium, with emissions allowed in all directions (solid); opaque inner medium, allowing only emissions which are outward in the $F F$ frame (dotted); and allowing only emissions which are outward in the $S$ frame (dashed-dotted). 

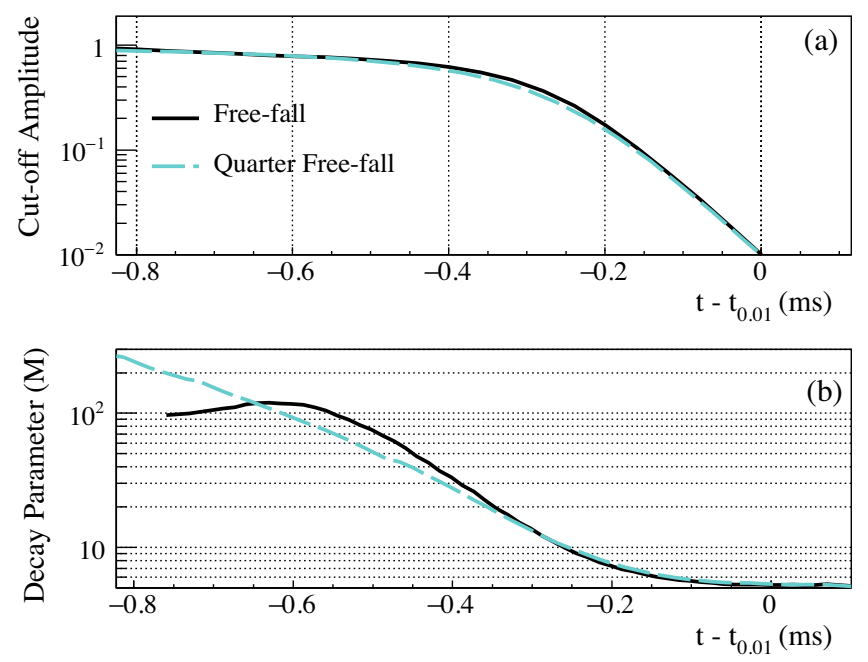

FIG. 8. (a) Luminosity and (b) decay parameter profiles for free-fall ( $f=1$, solid/black) and reduced ( $f=1 / 4$, dashed/blue) shell acceleration. The curves are shifted in time to meet at $t=t_{0.01}$, when the luminosity has fallen to $1 \%$ of its value at $t=0$.

and the velocity observed in the static frame $S$ will be

$$
\beta_{S}=\sqrt{\frac{C_{0}-C}{C_{0}+\left(f^{-1}-1\right) C}} .
$$

The resulting luminosity profile for $f=1 / 4$, corresponding to a doubling of the collapse time relative to the free fall case, is shown in Fig. 8. The slower velocity profile results in the extension of the slow drop by approximately $0.8 \mathrm{~ms}$ before the onset of the rapid decay. In order to compare the rapid decays, we shift the times so that the luminosity profiles meet where they have decreased to $1 \%$ of their starting value; the result is shown in Fig. 8. The quarter free fall decay parameter profile starts from a higher value and decreases more gradually throughout (the hump near the beginning of the free fall profile is due to the increasing contribution of inward trajectories). Once the rapid decay begins, however, it approaches a decay parameter $3 \sqrt{3} M$ in a manner very similar to the free-fall case.

Another variation of the opaque shell model is to allow the shell radius to fall at a speed different from that of the emitters themselves. In this case, the shell is defined by the radius below which the material is dense enough that the emitted neutrino is expected to scatter or be absorbed. The emitters, on the other hand, fall through this radius as they emit. The values of shell radii and emitter velocities as a function of time come from outside the present toy model, and here we use values from a GR1D [20] general relativistic hydrodynamic simulation, with modern neutrino transport and interaction rates, of a $40 M_{\odot}$ progenitor model [21] (with the Lattimer \& Swesty equation of state with $K_{0}=220 \mathrm{MeV}$ ), collapsing
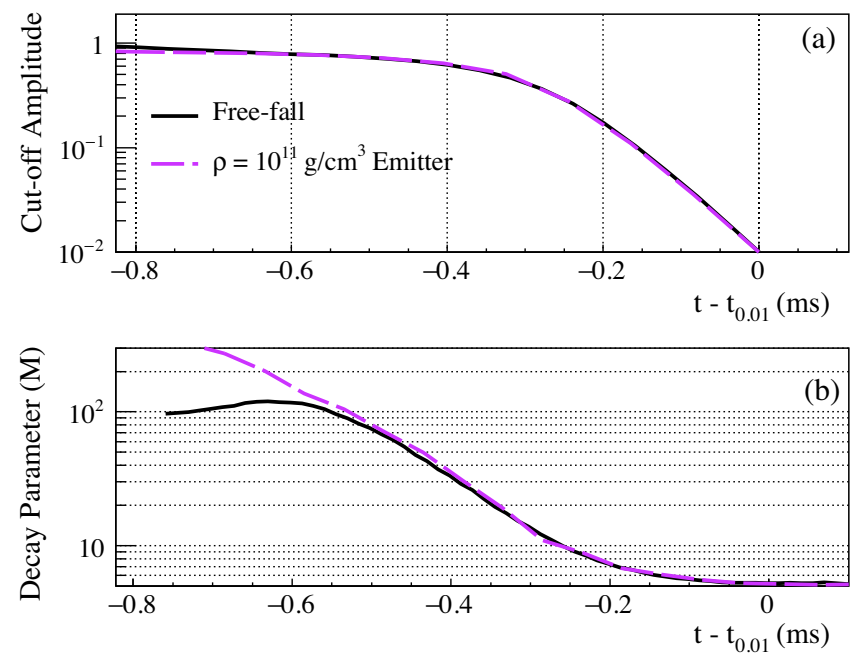

FIG. 9. (a) Luminosity and (b) decay parameter profiles for free falling and $\rho$-shell emitters. The curves are shifted in time to meet at $t=t_{0.01}$, when the luminosity has fallen to $1 \%$ of its value at $t=0$.

to a $2.25 M_{\odot}$ black hole [12]. The shell is defined by density $\rho=10^{11} \mathrm{~g} / \mathrm{cm}^{3}$, and simulations have been carried out until the shell has fallen from roughly $7 M$ to $3.5 M$, when the simulation ends; the maximum falling speed the shell attains is approximately $0.35 c$. When the simulation ends, neutrino emission is stopped, though emitted neutrinos which can escape are propagated to the observer. The resulting profiles are shown in Fig. 9. The luminosity profile remains flatter for longer when compared with the free fall case, but in the end the decay parameter still approaches $3 \sqrt{3} M$, the result of neutrinos emitted inwards (though not absorbed by the receding opaque shell) and subsequently trapped near radius $3 M$.

\section{TIME DELAYS IN THE KERR GEOMETRY}

In this section, we examine time delays in the Kerr geometry starting from the formulation of [22], which is summarized here. The metric in Boyer-Lindquist coordinates $(t, r, \theta, \phi)$ is

$$
\begin{aligned}
\mathrm{d} s^{2}= & -\left(1-\frac{2 M r}{\Sigma}\right) \mathrm{d} t^{2}-\frac{4 a M r \sin ^{2} \theta}{\Sigma} \mathrm{d} t \mathrm{~d} \phi+\frac{\Sigma}{\Delta} \mathrm{d} r^{2} \\
& +\Sigma \mathrm{d} \theta^{2}+\left(r^{2}+a^{2}+\frac{2 M r a^{2} \sin ^{2} \theta}{\Sigma}\right) \sin ^{2} \theta \mathrm{d} \phi^{2},
\end{aligned}
$$

where $J$ is the angular momentum of the black hole and

$$
\begin{gathered}
a \equiv \frac{J}{M} \\
\Delta \equiv r^{2}+a^{2}-2 M r
\end{gathered}
$$




$$
\Sigma \equiv r^{2}+a^{2} \cos ^{2} \theta
$$

The Kerr metric is axially symmetric, and, unlike in the Schwarzschild case, its geodesics do not in general lie in a plane. Geodesics are characterized by three constants: $E$ and $L$ of Eqs. (2) and (3), which are shared with the Schwarzschild case; and $Q$, the Carter constant [23], which can be said to characterize nonplanar motion. The equations of motion in these coordinates are

$$
\begin{aligned}
& \frac{\Sigma}{E}\left(\frac{\mathrm{d} t}{\mathrm{~d} \tau}\right)=\frac{1}{\Delta}(A-2 M r a b) \\
& \frac{\Sigma}{E}\left(\frac{\mathrm{d} \phi}{\mathrm{d} \tau}\right)=\frac{b}{\sin ^{2} \theta}+\frac{a}{\Delta}(2 M r-a b) \\
& \frac{\Sigma}{E}\left(\frac{\mathrm{d} \theta}{\mathrm{d} \tau}\right)=\sigma_{\theta} \sqrt{\Theta} \\
& \frac{\Sigma}{E}\left(\frac{\mathrm{d} r}{\mathrm{~d} \tau}\right)=\sigma_{r} \sqrt{R}
\end{aligned}
$$

with the useful shorthands

$$
\begin{aligned}
& \Theta \equiv q-\cos ^{2} \theta\left(\frac{b^{2}}{\sin ^{2} \theta}+a^{2}\left(\frac{\mu}{E^{2}}-1\right)\right) \\
& R \equiv\left(\left(r^{2}+a^{2}\right)-a b\right)^{2}-\Delta\left(\frac{\mu}{E^{2}} r^{2}+q+(a-b)^{2}\right) \\
& A \equiv\left(r^{2}+a^{2}\right)^{2}-a^{2} \Delta \sin ^{2} \theta,
\end{aligned}
$$

where $\mu=0$ gives null and $\mu=1$ timelike geodesics, and $\sigma_{r}$ and $\sigma_{\theta}$ indicate the direction of motion relative to the $r$ and $\theta$ axes. We define impact parameters $b \equiv L / E$, as before, and $q \equiv Q / E^{2}$. Radial directions coincide with $b=q=0$. We also identify corotating geodesics with $b>0$, and counter-rotating with $b<0$.

\section{A. Neutrino propagation time}

As with the nonrotating case, we model a thin shell of emitting matter falling freely from rest from an initial radius $r_{0}$. Instead of the frame of a static observer, we use the locally nonrotating frame (LNRF) [24]. An observer static in the LNRF is known as a zero angular momentum observer (ZAMO), as its four-velocity gives $L=0$.

The free-falling emitter in the Kerr geometry coincides with the ZAMO at $r=r_{0}$, at which location the emitter is initially at rest. Since, in the LNRF,

$$
\left(\frac{\mathrm{d} t}{\mathrm{~d} \tau}\right)_{L N R F}=\sqrt{\frac{A}{\Sigma \Delta}},
$$

one finds the constants of motion

$$
Q=a^{2}\left(1-E^{2}\right) \cos ^{2} \theta
$$

and

$$
E=\sqrt{\frac{\Sigma_{0} \Delta_{0}}{A_{0}}},
$$

where $\Sigma_{0}=\Sigma\left(r_{0}\right), \Delta_{0}=\Delta\left(r_{0}\right)$ and $A_{0}=A\left(r_{0}\right)$. In the coordinates of the distant observer,

$$
\frac{\mathrm{d} r}{\mathrm{~d} t}=\frac{\Delta}{\sqrt{A}} \cdot \sqrt{1-\frac{\Sigma \Delta / A}{\Sigma_{0} \Delta_{0} / A_{0}}}
$$

which in the LNRF becomes

$$
\left(\frac{\mathrm{d} r}{\mathrm{~d} t}\right)_{L N R F}=\sqrt{1-\frac{\Sigma \Delta / A}{\Sigma_{0} \Delta_{0} / A_{0}}} .
$$

These correspond to the velocities in Eqs. (5) and (7). For convenience in this section, we repurpose the subscript $S$ to represent the quantities observed in the LNRF. The velocity in Eq. (32) will therefore be denoted as $\beta_{S}$.

As before, we relate the emission angles in the freefalling frame $F F$ to constants of the neutrino's subsequent geodesic. The angle $\psi_{S}$ is defined relative to the outward radial direction in the LNRF,

$$
\left(\frac{\mathrm{d} r}{\mathrm{~d} t}\right)_{L N R F}=\cos \psi_{S}=\frac{\sigma_{r} \sqrt{R A}}{A-2 M r a b} .
$$

The corresponding angle $\psi_{F F}$ in the $F F$ frame can then be written in the same form as Eq. (9).

In the Kerr case, however, geodesics are not necessarily planar, and we define an out-of-plane angle $\eta$ as the azimuthal angle around the outward radial direction as the axis, with $\eta=0$ denoting the positive $\theta$ direction. In this way, $\eta \in[0, \pi)$ indicates a trajectory corotating with the black hole, and $\eta \in[\pi, 2 \pi)$ counterrotating. In the LNRF, this definition gives

$$
\left(r \frac{\mathrm{d} \theta}{\mathrm{d} t}\right)_{L N R F}=\sin \psi_{S} \cos \eta_{S}=\frac{\sigma_{\theta} \sqrt{\Theta \Delta A}}{A-2 M r a b},
$$

whereas in the $F F$ frame,

$$
\left(r \frac{\mathrm{d} \theta}{\mathrm{d} t}\right)_{F F}=\sin \psi_{F F} \cos \eta_{F F}=\frac{\sqrt{1-\beta_{S}^{2}} \sin \psi_{S} \cos \eta_{S}}{1+\beta_{S} \cos \psi_{S}} .
$$

Hence, for a given direction $\left(\psi_{F F}, \eta_{F F}\right)$ in the $F F$ frame, we calculate the quantities 


$$
\begin{aligned}
B & \equiv a^{2}-\frac{\Delta}{\sin ^{2} \theta} \\
\kappa^{2} & \equiv\left(v_{r}\right)_{S}^{2}+\left(v_{\theta}\right)_{S}^{2}=\cos ^{2} \psi_{S}+\sin ^{2} \psi_{S} \cos ^{2} \eta_{S} \\
\left(v_{r}\right)_{S} & =\cos \psi_{S}=\frac{\cos \psi_{F F}-\beta_{S}}{1-\beta_{S} \cos \psi_{F F}} \\
\left(v_{\theta}\right)_{S} & =\sin \psi_{S} \cos \eta_{S} \\
& =\frac{\sin \psi_{F F} \cos \eta_{F F}\left(1+\beta_{S} \cos \psi_{F F}\right)}{\sqrt{1-\beta_{S}^{2}}},
\end{aligned}
$$

from which we calculate the constants of the neutrino path

$b=\frac{A\left(2 M r a\left(1-\kappa^{2}\right)-\sigma_{b} \sqrt{\left(1-\kappa^{2}\right)\left(4 M^{2} r^{2} a^{2}-A B\right)}\right)}{A B-4 M^{2} r^{2} a^{2} \kappa^{2}}$,

where $\sigma_{b} \equiv b /|b|$ is the rotating direction relative to the rotation of the black hole, and

$$
q=\cos ^{2} \theta\left(\frac{b^{2}}{\sin ^{2} \theta}-a^{2}\right)+\sin ^{2} \psi_{S} \cos ^{2} \eta_{S} \frac{(A-2 M r a b)^{2}}{\Delta A} .
$$

We can now calculate the travel time in a manner similar to Eq. (10) using the integral

$$
T\left(b, q, r_{*} ; a, r_{E}\right)=\int_{r_{*}}^{r_{E}} \frac{A-2 M r a b}{\sigma_{r} \sqrt{R} \Delta} \mathrm{d} r .
$$

Since the integrand has an implicit dependence on $\theta$ via $A$, we propagate both $r$ and $\theta$ along the geodesic by equating integrals of the last two equations of Eq. (26):

$$
\int_{r_{*}}^{r} \frac{\mathrm{d} r}{\sigma_{r} \sqrt{R}}=\int_{\theta_{*}}^{\theta} \frac{\mathrm{d} \theta}{\sigma_{\theta} \sqrt{\Theta}}
$$

For null geodesics, the $\theta$ integral is evaluated with

$$
\int_{\theta_{*}}^{\theta} \frac{\mathrm{d} \theta}{\sqrt{\Theta}}=\int_{\cos \theta_{*}}^{\cos \theta} \frac{-\mathrm{d} \cos \theta}{\sqrt{q+\left(a^{2}-q-b^{2}\right) \cos ^{2} \theta-a^{2} \cos ^{4} \theta}} .
$$

The time delays are calculated in the same fashion as Eqs. (12) and (13), albeit with an extra dependence on $q$. For the inward case, the periapsis is the largest real root of $R$ at some given $b$ and $q$.

The escape conditions for the Kerr metric are more complicated than those for the Schwarzschild metric and entail a number of cases which are tabulated in [25] for a full Kerr space and [22] for a disc model.

\section{B. Luminosity profile}

Following the same procedure for deriving the gravitational redshift in the Schwarzschild case, we find the gravitational redshift to be

$$
\frac{\nu_{E}}{\nu_{S}}=\sqrt{\frac{\Sigma \Delta}{A}},
$$

where $\nu_{E}$ and $\nu_{S}$ are the energies observed on Earth and in the LNRF at the emission position. The total redshift factor is then

$\zeta\left(b, q, r_{*}, \theta_{*}, \sigma_{r}\right)=\frac{\nu_{E}}{\nu_{*}}=\sqrt{\frac{\Sigma \Delta}{A}} \times \frac{\sqrt{1-\beta_{S}^{2}}}{1+\beta_{S} \cos \psi_{S}}$,

where $\nu_{*}$ is the neutrino energy in the $F F$ frame of the emitter.
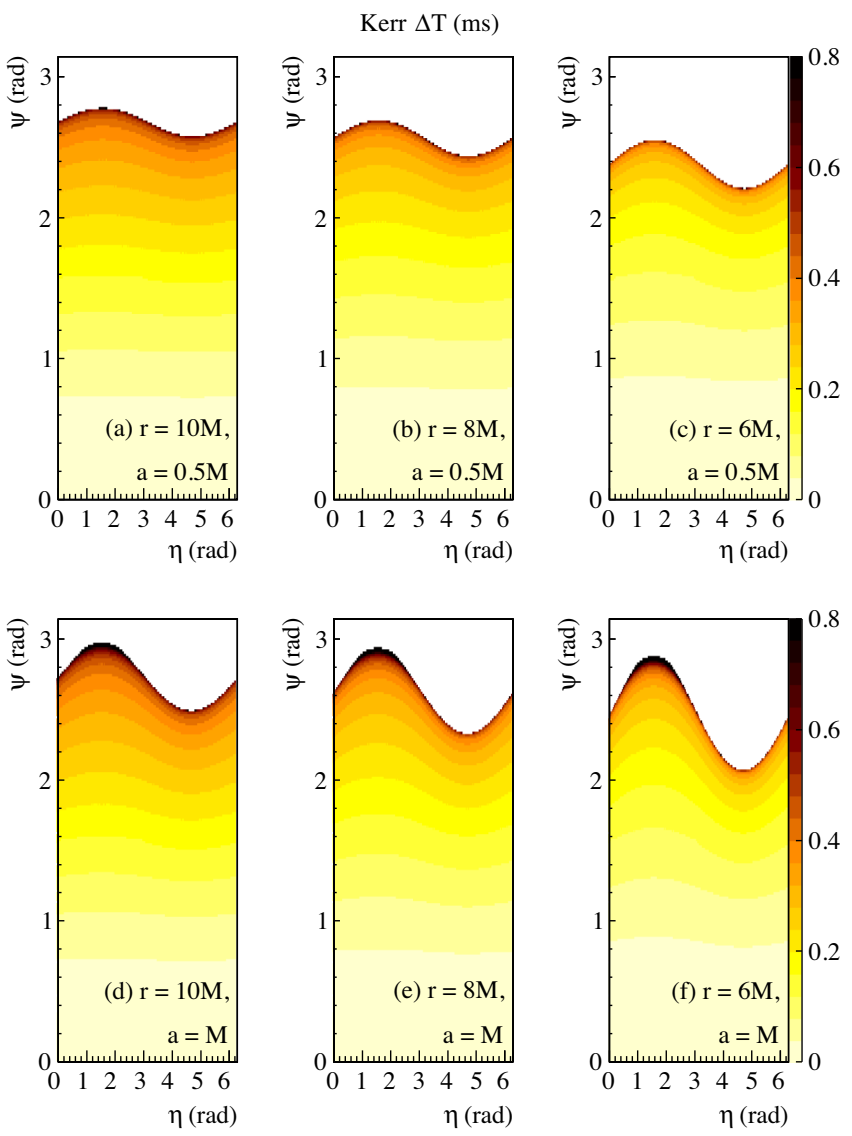

FIG. 10. Time delays (color or gray scale, in units of milliseconds), relative to that of the radial direction, as a function of the emission direction in the $F F$ frame, at different emission radii for a collapsing ring around a rotating black hole. The white region at large $\psi_{F F}$ indicates nonescaping directions; in some areas the black region, which always borders on a white region, is too narrow to show up in the figure. The black hole mass is $M=2.5 M_{\odot}$, and the ring starts from rest at $r_{0}=10 M$. Top row: subextremal rotation $a=0.5 M$. Bottom row: extremal rotation $a=M$. 


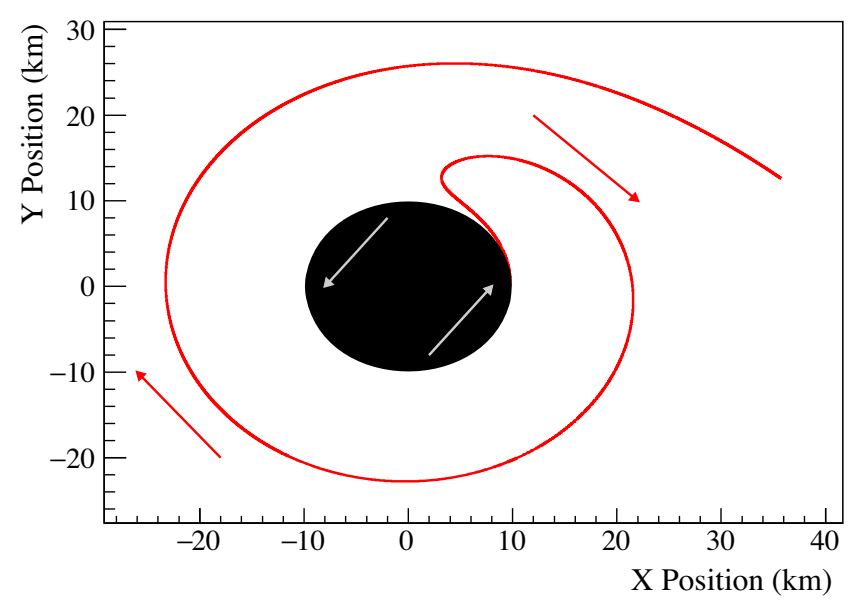

FIG. 11. An example geodesic for a particle emitted close to the event horizon of a rotating black hole. This geodesic is classified as "counterrotating" due to strong local frame dragging near the horizon, i.e., the trajectory is opposite the direction of rotation in its LNRF, though not in the coordinate system of a distant observer.

We use the same ray-tracing Monte Carlo approach as before. In order to reduce the simulation time, however, we only calculate results for emissions from a contracting ring of matter in the equatorial plane. Figure 10 shows the time delays (relative to that of radial emission from the given radius) as a function of $\psi_{F F}$ and $\eta_{F F}$, for rotation parameters $a=0.5 M$ and $a=M$. The rotation in each case is in the direction of increasing azimuthal angle $\phi$. Rotation introduces an asymmetry in $\eta_{F F}$ : at a given $\psi_{F F}$, counterrotating geodesics, with $\eta_{S} \in(\pi, 2 \pi)$, tend to undergo longer delays (note that the white regions in Fig. 10 indicate trajectories with above-critical impact parameters, i.e., diverging escape times). The delay is especially long for initially counter-rotating geodesics reversing direction (a) Redshift Curve

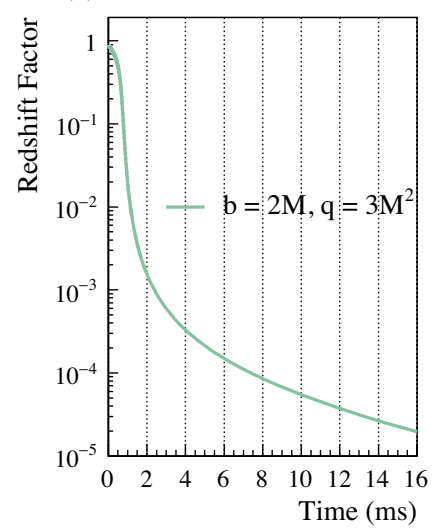

(b) Decay Parameter

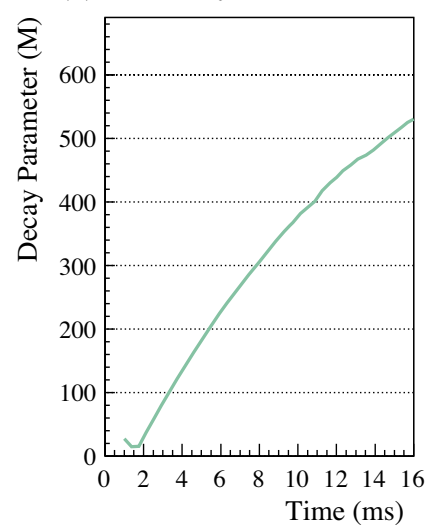

FIG. 12. (a) Redshift factor and (b) redshift decay parameter of emissions with $b=2 M$ and $q=3 M^{2}$, for orbits near the horizon at $r=M$, plotted against observation time for a $2.5 M_{\odot}$ extremal $(a=M)$ Kerr black hole.

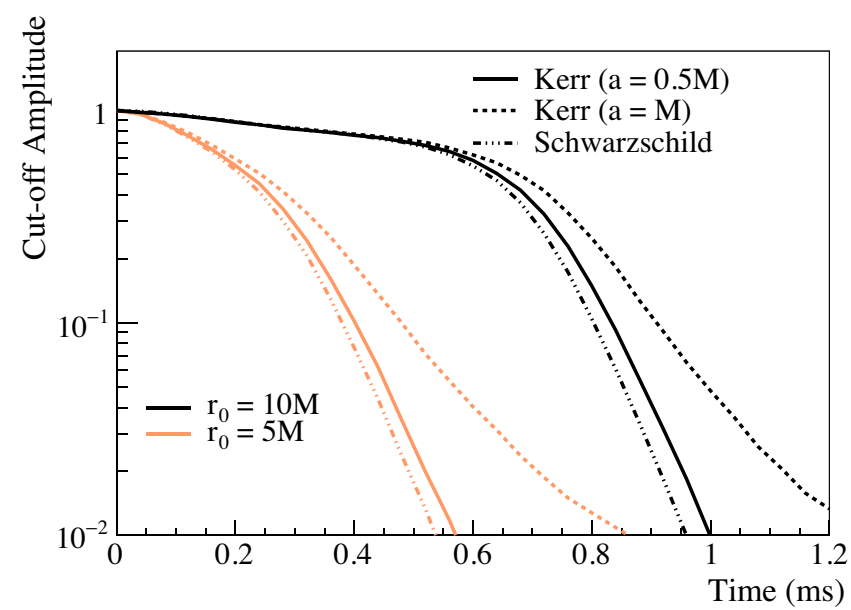

FIG. 13. Luminosity profiles of the neutrino cutoff in the Kerr geometry, with $a=0.5 M$ and $a=M$, and at different initial radii $r_{0}=5 M$ and $10 M$.

before escaping, as illustrated in one example in Fig. 11. A further example of the effect of such geodesics is shown in Fig. 12, which shows the redshift curve and decay parameter, as a function of observation time, for emissions with $b=2 M$ and $q=3 M^{2}$ originating near the horizon at $r=M$ of an extremal Kerr black hole. As seen in the decay parameter curve, such geodesics "leak" out very slowly compared to the usual cutoff timescale of $O(0.1) \mathrm{ms}$ in a manner similar to the leakage from the Schwarzschild black hole's "photon sphere" at $r=3 M$.

The resulting cutoff profile is shown in Figs. 13 and 14. The long tail is not very evident in the subextremal case of $a=0.5 M$, but in the extremal case of $a=M$, the modification of the cutoff is more significant. It is also evident

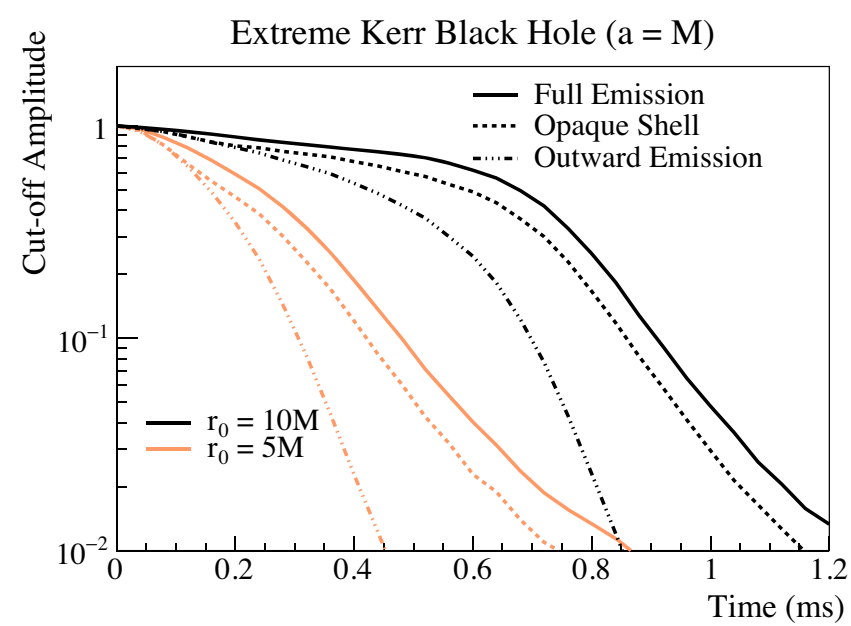

FIG. 14. The three inward emission scenarios in an extreme Kerr black hole: transparent medium, with emissions allowed in all directions (solid); opaque inner medium, allowing only emissions which are outward in the $F F$ frame (dotted); and allowing only emissions which are outward in the LNRF, or $S$ frame (dashed-dotted). 
from Fig. 14 that the modification is mostly due to those geodesics which are emitted inwards in the LNRF, but are outward in the $F F$ frame. Unlike in the nonrotating case, the decay parameter of the tail does not approach a limiting value, but rather continues to increase in a manner which may be noticeable even before the time the neutrinos are redshifted below detectable energies.

\section{SUMMARY AND DISCUSSION}

We have investigated the contribution of nonradial neutrino emissions to the shape of the neutrino cutoff expected upon the formation of a black hole within a corecollapse supernova. Our toy calculations, based on raytracing null geodesics from contracting matter shells or rings, show for the Schwarzschild case the cutoff starting with a slow decrease in luminosity over several tenths of milliseconds, depending on the model of the shell and emitters' velocities, followed by a rapid decrease which approaches an exponential decay with time constant $3 \sqrt{3} M$, a value calculated in the 1960 s by Podurets [4] and Ames and Thorne [5]. The $3 \sqrt{3} M$ time constant features in the luminosity profiles across different modifications to the model, indicating that all such models end up with neutrinos slowly leaking from near the radius $3 M$. If this part of the cutoff can be resolved in time, it would represent an independent handle on the mass of the newly formed black hole.

We estimate how many neutrino events may be available for resolving the time constant by integrating a simple exponential tail, as if the cutoff begins abruptly rather than turning over a "knee" as seen in Fig. 7 and elsewhere. We use the $40 M_{\odot}$ model from Sec. IIIC, observed at a distance of $10 \mathrm{kpc}$. Event rates for several neutrino detectors can be found in Fig. 3 of [26]. Super-Kamiokande [27] and JUNO [28] may be expected to see an event rate of around 15 per ms before the cutoff, followed by a tail of approximately 0.7 events. This yield is unlikely to result in a measurement but is large enough that it introduces a systematic uncertainty in how well the cutoff can be localized in time; this uncertainty would then feed into applications such as using the observed cutoff in several detectors to triangulate the direction to the CCSN. On the other hand, the estimated event rate for Hyper-Kamiokande [29] before the cutoff is around 100 events per ms, followed by a tail of 5 events, which may indeed give a crude value even after taking into account the uncertainty in when the exponential cutoff begins. It should be noted, however, that these events likely will be mixed with those of other effects, among them an "echo" of neutrinos scattering off infalling material which is explored in a companion work [26].

In the Kerr case, we see that even for rotations as large as $a=0.5 M$, the nonradial geodesics appear to introduce a small delay to the cutoff, but do not otherwise noticeably modify the tail or its time constant. For extreme rotation, however, the neutrino leakage from near the horizon extends the tail significantly, and doubles the number of events expected to be observed. Even though the extreme rotation case is usually considered to be unlikely, it highlights the desirability of resolving the shape of the cutoff, not just to measure rotation, but also to gauge the validity of using the time constant as a mass measurement.

Even though this study is very simplistic, it suggests that if there is an abrupt drop in the neutrino emission from a CCSN, signaling the formation of a black hole, it is worth examining the shape of the cutoff in more detail from both theoretical and observational perspectives. The advent of the next generation's larger detectors, as well as the combination of detectors via SNEWS, puts such measurements tantalizingly within reach.

\section{ACKNOWLEDGMENTS}

The authors would like to thank Philipp Podsiadlowski, Shuai Zha, Ming-chung Chu, Luc Nguyen, Steve Biller, Armin Reichold, and the Oxford SNO + group for stimulating and useful discussions. This research is supported by the Science and Technology Facilities Council of the United Kingdom (Grant No. ST/S000933/1) and the Swedish Research Council (Project No. 2020-00452).
[1] S. Al Kharusi et al. (SNEWS Collaboration), SNEWS 2.0: A next-generation supernova early warning system for multi-messenger astronomy, New J. Phys. 23, 031201 (2021).

[2] A. Burrows and D. Vartanyan, Core-collapse supernova explosion theory, Nature (London) 589, 29 (2021).

[3] J. B. Hartle, Gravity: An Introduction to Einstein's General Relativity (Addison-Wesley, San Francisco, 2003).

[4] M. A. Podurets, Asymptotic behavior of the optical luminosity of a star in gravitational collapse, Astr. Zh. 41, 1090 (1964) [M. A. Podurets, Sov. Astron. 8, 868 (1965)].
[5] W. L. Ames and K. S. Thorne, The optical appearance of a star that is collapsing through its gravitational radius, Astrophys. J. 151, 659 (1968).

[6] J. F. Beacom, R. N. Boyd, and A. Mezzacappa, Technique for Direct eV Scale Measurements of the $\mathrm{Mu}$ and Tau Neutrino Masses using Supernova Neutrinos, Phys. Rev. Lett. 85, 3568 (2000).

[7] J. F. Beacom, R. N. Boyd, and A. Mezzacappa, Black hole formation in core-collapse supernovae and time-of-flight measurements of the neutrino masses, Phys. Rev. D 63, 073011 (2001). 
[8] T. W. Baumgarte, S. A. Teukolsky, S. L. Shapiro, H. T. Janka, and W. Keil, Delayed collapse of hot neutron stars to black holes via hadronic phase transitions, Astrophys. J. 468, 823 (1996).

[9] J. F. Beacom and P. Vogel, Can a supernova be located by its neutrinos?, Phys. Rev. D 60, 033007 (1999).

[10] V. Brdar, M. Lindner, and X.-J. Xu, Neutrino astronomy with supernova neutrinos, J. Cosmol. Astropart. Phys. 04 (2018) 025.

[11] L. Sarfati, R. S. L. Hansen, and I. Tamborra, Triangulating black hole forming stellar collapses through neutrinos, arXiv:2110.02347.

[12] E. O'Connor, An open-source neutrino radiation hydrodynamics code for core-collapse supernovae, Astrophys. J. Suppl. Ser. 219, 24 (2015).

[13] L. Walk, I. Tamborra, H.-T. Janka, A. Summa, and D. Kresse, Neutrino emission characteristics of black hole formation in three-dimensional simulations of stellar collapse, Phys. Rev. D 101, 123013 (2020).

[14] K. Lake and R. C. Roeder, Note on the optical appearance of a star collapsing through its gravitational radius, Astrophys. J. 232, 277 (1979).

[15] S. L. Shapiro, Thermal radiation from stellar collapse to a black hole, Phys. Rev. D 40, 1858 (1989).

[16] S. V. Dhurandhar and C. V. Vishveshwara, Neutrinos in gravitational collapse. I-Analysis of trajectories, Astrophys. J. 245, 1094 (1981).

[17] S. V. Dhurandhar and C. V. Vishveshwara, Neutrinos in gravitational collapse: Analysis of the flux profile, Pramana 22, 159 (1984).

[18] S. W. Li, L. F. Roberts, and J. F. Beacom, Exciting prospects for detecting late-time neutrinos from core-collapse supernovae, Phys. Rev. D 103, 023016 (2021).
[19] S. Chandrasekhar, The Mathematical Theory of Black Holes (Oxford University Press, New York, 1983).

[20] E. O'Connor and C.D. Ott, A new open-source code for spherically symmetric stellar collapse to neutron stars and black holes, Classical Quant. Grav. 27, 114103 (2010).

[21] S. E. Woosley and A. Heger, Nucleosynthesis and remnants in massive stars of solar metallicity, Phys. Rep. 442, 269 (2007).

[22] T. Igata, K. Kohri, and K. Ogasawara, Photon emission from inside the innermost stable circular orbit, Phys. Rev. D 103, 104028 (2021).

[23] B. Carter, Global structure of the Kerr family of gravitational fields, Phys. Rev. 174, 1559 (1968).

[24] J. M. Bardeen, W. H. Press, and S. A. Teukolsky, Rotating black holes: Locally nonrotating frames, energy extraction, and scalar synchrotron radiation, Astrophys. J. 178, 347 (1972).

[25] K. Ogasawara and T. Igata, Complete classification of photon escape in the Kerr black hole spacetime, Phys. Rev. D 103, 044029 (2021).

[26] S. Gullin, E. O'Connor, J.-S. Wang, and J. Tseng, Neutrino echos following black hole formation in core-collapse supernovae, submitted to AAS journals, arXiv:2109 .13242 .

[27] Y. Fukuda et al. (Super-Kamiokande Collaboration), The Super-Kamiokande detector, Nucl. Instrum. Methods Phys. Res., Sect. A 501, 418 (2003).

[28] F. An et al. (JUNO Collaboration), Neutrino physics with JUNO, J. Phys. G 43, 030401 (2016).

[29] K. Abe et al. (Hyper-Kamiokande Collaboration), HyperKamiokande design report, arXiv:1805.04163. 